\title{
Comparación Microscópica del Barro Dentinario Resi- dual en Conductos Radiculares, tras Instrumentación Rotatoria con y sin un Quelante Viscoso
}

\author{
A Microscopic Comparison of the Residual Smear Layer Remaining in Root \\ Canals, after Rotary Instrumentation with and without a Viscous Chelator
}

Gastón Zamora*; Rodrigo Fuentes"; Erika Peschke" \& Felipe Nenen ${ }^{*+*+*}$

ZAMORA, G.; FUENTES, R.; PESCHKE, E. \& NENEN, F. Comparación microscópica del barro dentinario residual en conductos radiculares, tras instrumentación rotatoria con y sin un quelante viscoso. Int. J. Odontostomat., 5(2):165-170, 2011.

RESUMEN: Quelantes de consistencia viscosa son utilizados para ayudar la instrumentación en conductos muy estrechos, no obstante, podrían contribuir al acúmulo de más barro dentinario. El objetivo de este estudio fue comparar el barro dentinario residual tras instrumentar conductos radiculares, con y sin la utilización de un quelante viscoso. 39 incisivos inferiores extraídos fueron instrumentados con Protaper ${ }^{\circledR}$ y divididos en tres grupos. La irrigación de los grupos A y B fue con $\mathrm{NaClO} 5,25 \%$ entre cada lima. Al terminar la instrumentación se irrigó con EDTA 17\% (1 minuto), luego con NaClO $5,25 \%$ y finalmente con suero fisiológico. Además, en el grupo A se utilizó un quelante viscoso entre cada lima. El grupo C sólo fue irrigado con suero fisiológico. Tres dientes no fueron instrumentados e irrigados (control negativo). Las raíces fueron seccionadas longitudinalmente y divididas en tercios para ser evaluadas bajo microscopía óptica. Los valores de detritus obtenidos fueron analizados con estadística no paramétrica $(p<0,05)$. En cada tercio medio se encontraron valores muy bajos de barro dentinario, siendo iguales en todos los grupos. En cambio en el tercio apical de los grupos A y $\mathrm{C}$ se encontró significativamente más barro dentinario que en el grupo B. Conductos instrumentados con la ayuda de un quelante viscoso, acumularon significativamente más barro dentinario.

PALABRAS CLAVE: quelante viscoso, barro dentinario, instrumentación rotatoria.

\section{INTRODUCCIÓN}

El objetivo de la instrumentación del canal radicular es configurar y limpiar el sistema de canales radiculares. El concepto de limpieza incluye la remoción de la dentina infectada y del tejido orgánico, a través de la instrumentación e irrigación. Todos los instrumentos endodónticos crean detritus y barro dentinario como consecuencia de su accionar sobre las paredes del canal radicular (Jeon et al., 2003). El detritus está compuesto por virutas de dentina y/o tejido residual vital o necrótico adheridos a la pared del canal radicular (Hülsmann et al., 1997). La Asociación Americana de Endodoncistas (2003), definió el barro dentinario, como una película de detritus retenido sobre la dentina u otra superficie. Si bien, es aún controversial la influencia del barro dentinario en el éxito del tratamiento endodóntico, la evidencia nos indica que su presencia puede disminuir la penetración de la medicación e irrigantes en los túbulos dentinarios infectados. Lo anterior, puede llegar a convertirse en un obstáculo para el óptimo sellado del canal radicular, aumentado con ello el riesgo de microinfiltración del relleno endodóntico (Shahravan et al., 2007). Además, el barro dentinario infectado contiene bacterias y tejido necrótico, el cual puede actuar como sustrato para las bacterias, permitiéndoles que penetren aun más en los túbulos dentinarios (George et al., 2005), siendo deseable su remoción debido al efecto perjudicial (Lim et al., 2003).

\footnotetext{
Profesor Titular. Cátedra de Endodoncia, Facultad de Odontología, Universidad de Valparaíso, Chile.

* Profesor Auxiliar. Cátedra de Patología y Diagnóstico Oral, Facultad de Odontología, Universidad de Valparaíso, Chile.

*** Cirujano Dentista EDF, Hospital Los Vilos. Servicio de Salud de Coquimbo, Chile.

**** Cirujano Dentista, Sanidad Dental, Armada de Chile.
} 
La instrumentación por sí sola no es capaz de producir una limpieza de las paredes del canal. En este sentido, la irrigación juega un rol importante en el desbridamiento y la remoción del barro dentinario durante la preparación. El hipoclorito de sodio $(\mathrm{NaClO})$ es una solución irrigadora ampliamente usada en los tratamientos del canal radicular por sus propiedades antibacterianas y habilidad de disolver tejido orgánico, sin embargo, no es efectivo en la remoción de la parte inorgánica del barro dentinario (Naenni et al., 2004). El ácido etilenendiaminotetracético (EDTA) es un agente quelante que puede remover la parte inorgánica del barro dentinario. Así, la combinación de $\mathrm{NaClO}$ y EDTA ha sido reportada, como la más adecuada para la remoción del barro dentinario (Grandini et al., 2002). Glyde $®$ es un acondicionador del canal radicular que contiene EDTA y peróxido de carbamida en una base hidrosoluble. De acuerdo al fabricante, éste se debe usar en conjunto con $\mathrm{NaClO}$, como complemento durante la instrumentación. Este quelante viscoso facilita la preparación del canal radicular durante la conformación, debido al efecto del EDTA y a la efervescencia del oxígeno liberado del peróxido de carbamida, permitiendo, en teoría, remover el detritus fácilmente. En la búsqueda de estrategias óptimas para el tratamiento del canal radicular, las acciones químicas básicas y las interacciones de las sustancias que se utilizan han sido descuidadas en gran medida. Por ejemplo, la disolución del tejido necrótico y la acción antimicrobiana del hipoclorito de sodio son únicamente funciones del cloro disponible en solución (Zehnder et al., 2002). El cloro disponible en solución de $\mathrm{NaClO}$ es casi en su totalidad en forma de hipoclorito (OCl),

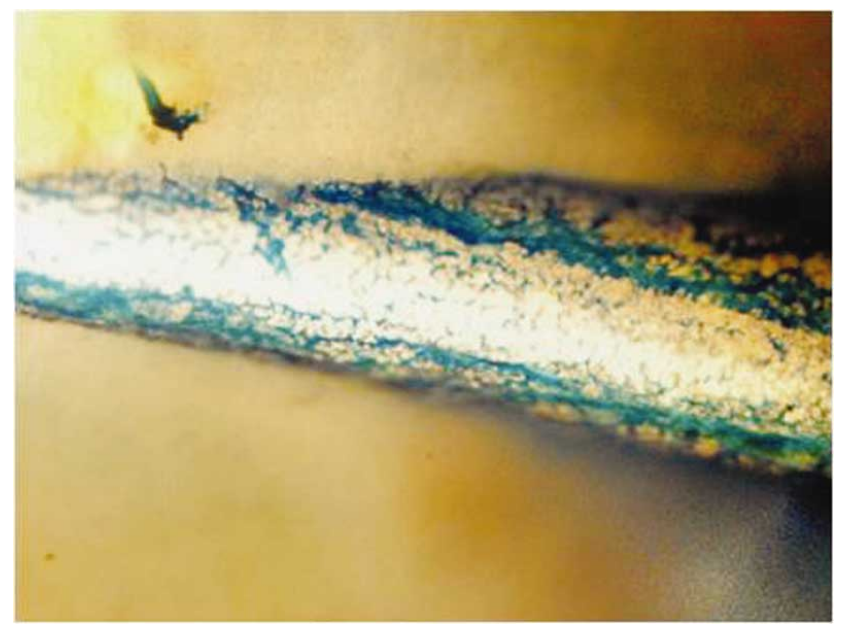

Fig. 1. Pared de un conducto radicular sin instrumentar ni irrigar observada al microscopio óptico. Es posible observar el entradamado de los restos del tejido pulpar teñido y la ausencia de detritus y barro dentinario. que es un agente extremadamente reactivo que interactúa fácilmente con cualquier compuesto que pueda ser oxidado. EI EDTA en solución acuosa de inmediato interfiere con la capacidad de disolver tejido orgánico y con la acción antibacteriana de la solución del hipoclorito (Grawehr et al., 2003; Zehnder et al., 2005). Al mismo tiempo, la incorporación de peróxido en pastas quelantes que son utilizadas en conjunto con hipoclorito, también parece cuestionable. Como el hipoclorito es un agente oxidante más fuerte que el peróxido, éste puede ser reducido por el peróxido y perder su eficacia (Girard et al., 2005).

Esta investigación tiene como objetivo comparar microscópicamente el barro dentinario residual, al instrumentar conductos radiculares con instrumentación rotatoria, con y sin aplicación de Glyde®

\section{MATERIAL Y MÉTODO}

Preparación de las muestras. 39 dientes recolectados fueron desinfectados en una solución de $\mathrm{NaClO}$ al $5,25 \%$ por 10 minutos y luego fueron lavados con suero fisiológico. La corona fue eliminada, realizando una muesca a nivel de la unión amelocementaria; para ello, se empleó una pieza de mano con un disco de acero y luego con cincel y martillo se separaron ambas partes. La permeabilidad de los conductos se comprobó introduciendo una lima K 10 (Maillefer $尺$ ), hasta que su punta fuera visible más allá del foramen apical. Luego, los dientes seccionados fueron almacenados en recipientes individuales y distribuidos aleatoriamente en 3 grupos de 12 unidades; un cuarto grupo (3 dientes seccionados) sirvió de control negativo, los cuales no fueron instrumentados ni irrigados (Fig. 1).

\section{Instrumentación químico-mecánica de los conduc- tos radiculares.}

Grupo A: Los conductos fueron instrumentados con el sistema Protaperâ alternado con Glyde $®$ de acuerdo al protocolo del fabricante, empleando un motor XSmart@ (Dentsply). Primero, se aplicó Glyde $®$ en la entrada del conducto, y luego con la lima $\mathrm{S} 1$ se realizó la preparación de los $2 / 3$ coronarios del conducto, con movimientos de entrada y salida. A continuación, se calculó la longitud de trabajo (LT) del conducto, sustrayéndole $1 \mathrm{~mm}$ a la longitud real del conducto desde el borde cervical hasta el foramen. Después se instrumentó con la siguiente secuencia de limas: S1, 
S2, F1, F2 y F3 a LT, siempre con movimientos de pincelado. En caso que existiera alguna resistencia para obtener LT, se recurrió a la lima SX para vencer dicho obstáculo. Cada vez que se utilizó una lima Protaperâ, está fue recubierta con Glyde ${ }^{\circ}$.

Grupo B: Los conductos fueron preparados siguiendo el mismo método que en el grupo A, pero sin utilizar Glyde®. Entre cada instrumento utilizado en los grupos $\mathrm{A}$ y $\mathrm{B}$, se recapituló con una lima $\mathrm{K} 10$ y se irrigó con $2 \mathrm{ml}$ de $\mathrm{NaClO}$ al 5,25\%, con una jeringa hipodérmica $30 \mathrm{G}$ calibrada a $3 \mathrm{~mm}$ de la LT. La irrigación final en los grupos A y B, se ejecutó con $2 \mathrm{ml}$ de EDTA al $17 \%$ por 1 minuto, luego $2 \mathrm{ml}$ de $\mathrm{NaClO}$ al 5,25\%, seguidos de $4 \mathrm{ml}$ de suero fisiológico. Por último, cada conducto fue secado con tres conos de papel \#30.

Grupo C (control positivo): Los conductos fueron instrumentados con el sistema Protaperâ siguiendo el mismo protocolo, pero la irrigación usada durante la recapitulación, sólo se realizó con $2 \mathrm{ml}$ de suero entre cada instrumento. Al terminar la instrumentación se emplearon $4 \mathrm{ml}$ de irrigación con suero. Finalmente, cada conducto fue secado con tres conos de papel \#30.

Cabe destacar que cada set de limas Protaperâ fue utilizado sólo 3 veces, dentro de un mismo grupo.

Evaluación de las muestras. En cada diente seccionado, se realizó con discos de acero montados en una pieza de mano un surco longitudinal y dos perpendiculares al eje del mismo. Lo anterior, permitió dividir la raíz en 3 tercios iguales. Luego, las caras proximales de las raíces fueron desgastadas manualmente contra una piedra de Arkansas, de tal manera dejar una superficie plana y delgada para la observación. Además, con un cincel y un martillo cada raíz fue separada horizontalmente en tres partes. En seguida, los tercios medio y apical, fueron divididos longitudinalmente en dos. Sólo la mitad más integra de cada tercio fue escogida. Finalmente, los especímenes fueron teñidos con azul de metileno al $1 \%$ durante 2 minutos antes de ser lavados con solución salina al 0,9\%. Las muestras fueron observadas en microscopio óptico por dos examinadores independientes, quienes desconocían a cual grupo pertenecía cada espécimen. Para evaluar las muestras se aplicó la escala de medición que emplearon Lim et al., en el cual asignaban la puntuación 1 cuando la pared del conducto radicular se encontraba limpia o con muy poco detritus (Fig. 2); 2 cuando había presencia de detritus hasta en la mitad del canal (Fig. 3); y 3 cuando existía presencia de detritus en más de la mitad del canal radicular (Fig. 4). Luego, los datos fueron analizados con estadística no paramétrica en el software estadístico Stata 11.0, utilizando un valor $\mathrm{a}=0,05$ y un valor $b=0,1$.

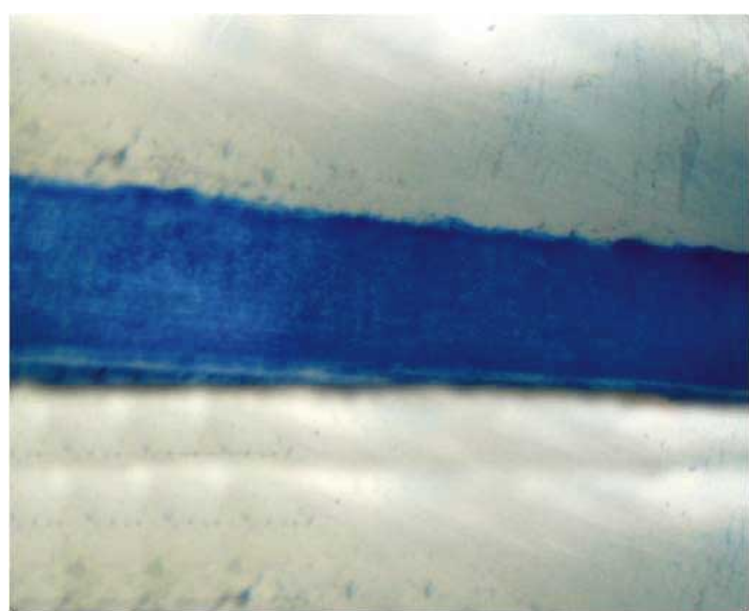

Fig. 2. Pared de un conducto radicular con ausencia de detritus (puntuación 1), al ser observada al microscopio óptico.

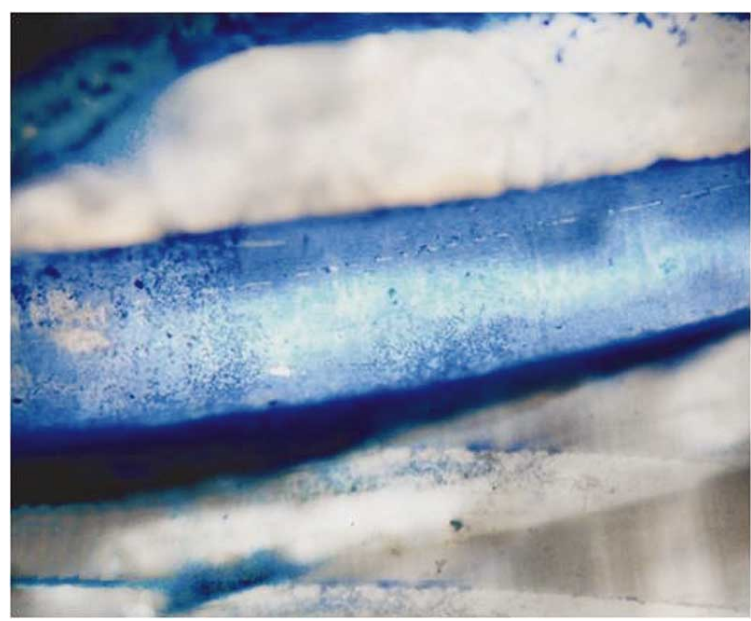

Fig. 3. Conducto radicular con menos de la mitad de la pared con detritus (puntuación 2), al ser observada al microscopio óptico.

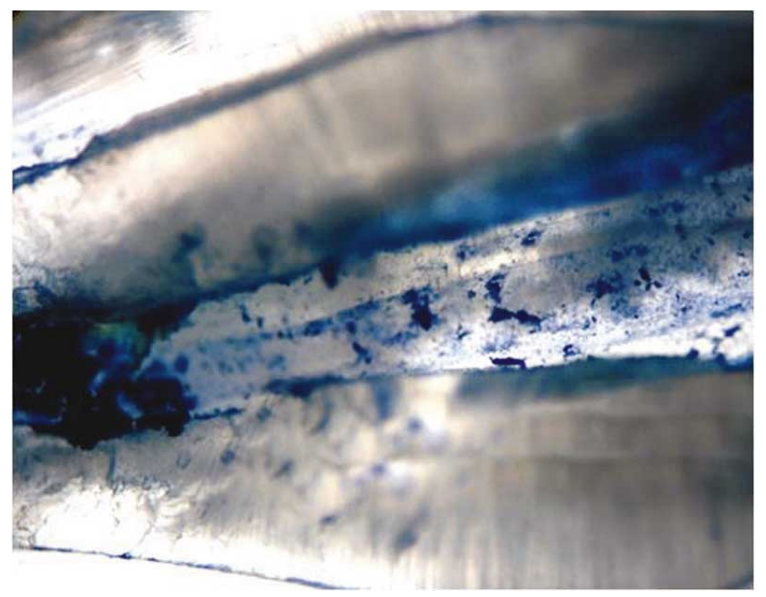

Fig. 4. Conducto radicular con más de la mitad de la pared con detritus (puntuación 3), al ser observada al microscopio óptico. 


\section{RESULTADOS}

El promedio de valores para la reproductibilidad intraobservador e interobservador, fue superior a 0,9 indicando una alta concordancia.

En el tercio medio se observó que en todos los grupos predominó la puntuación 1, seguida por la puntación 2 (Fig. 5). En cuanto al tercio apical, es posible apreciar que la puntuación que se asignó al grupo donde se utilizó Glyde $囚$, fue mayoritariamente 2, en cambio en el grupo donde este quelante viscoso no fue empleado, la puntuación preferente fue 1. Para el grupo control positivo, la puntuación asignada arrojo igual cantidad para 2 y 3 (Fig. 6).

De acuerdo a la metodología planteada, se determinó que entre los tercios medios de los distintos gru-

\section{Tercio medio}

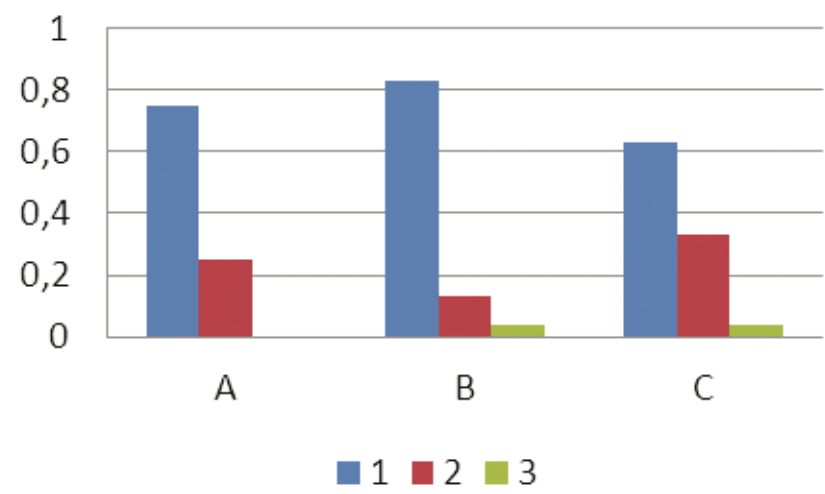

Fig. 5. Proporción de puntuación de los grupos de estudio en el tercio medio.

\section{Tercio apical}

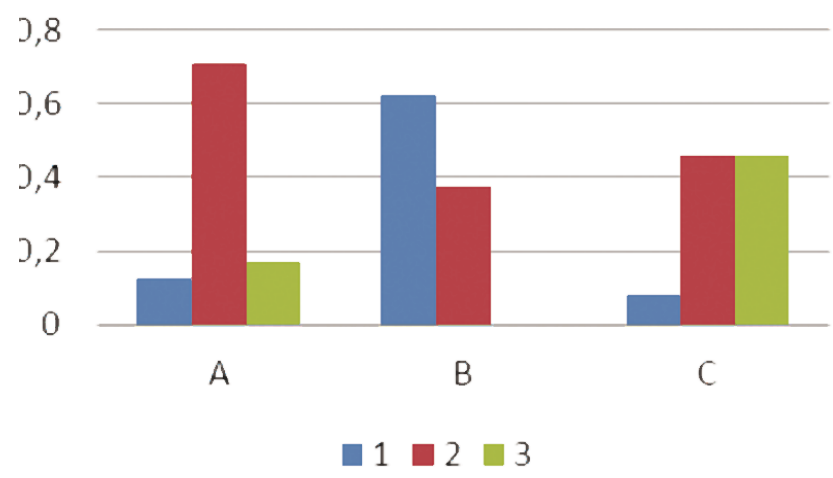

Fig. 6. Proporción de puntuación de los grupos de estudio en el tercio apical. pos, no existieron diferencias (test de Kruskall Wallis, $p=0,5869$ ), situación distinta a lo observado entre los tercios apicales donde sí existieron diferencias significativas (test de Kruskall Wallis, $p=0,0007$ ).

A nivel del tercio apical, se observó que en el grupo donde se utilizó Glyde ${ }^{\circledR}$ hubo significativamente más barro dentinario que en el grupo donde no se utilizó (test de Mann Whitney, $p=0,0035)$. Por otra parte, no existieron diferencias significativas entre el grupo control positivo y aquel que utilizó este quelante viscoso $(p=0,1430)$.

Al momento de comparar los tercios medios con los apicales en un mismo grupo, se encontraron diferencias significativas en el grupo $A$ y $C$ (test de Wilcoxon, $p<0,02)$, pero no así en el grupo $B(p=0,1797)$.

\section{DISCUSIÓN}

Independiente del uso de quelante viscoso, en los tercios medios se encontraron canales muy limpios al ser observados microscópicamente. Estudios anteriores reportaron que la eliminación del barro dentinario en los tercios medios y coronales fue muy efectiva con y sin la utilización de un quelante viscoso (Grandini et al.)

Distintos resultados hemos encontrado en el tercio apical. Así, en aquel grupo donde se utilizó el quelante viscoso, se observó significativamente más detritus, comparado con aquel grupo que no lo usó. Los reportes hasta ahora han sido contradictorios referentes al tema. Hülsmann et al. (2002), por ejemplo, llegó a las mismas conclusiones que nuestro estudio, en cambio otros investigadores, a pesar de encontrar más detritus y barro dentinario en el tercio apical al irrigar con un quelante viscoso, las diferencias no fueron estadísticamente significativas (Lim et al.; Dotto et al., 2007). Esto último puede deberse al mayor diámetro apical alcanzado durante la instrumentación, mejorando la penetración de los irrigantes, con lo que es posible remover más eficientemente detritus y barro dentinario (Baugh \& Wallace, 2005).

Cuando se comparó el tercio medio y apical de un mismo grupo, se encontró que en el grupo donde no se utilizó el quelante viscoso, no existieron diferencias significativas. Resultados distintos han sido reportados en investigaciones donde siempre se encontró que el tercio apical generaba mayor cantidad de barro dentinario, independiente del uso de un quelante viscoso o no (Prati et al., 2004; Yang et al., 2008). 
La razón de los resultados obtenidos en el tercio apical al utilizar este quelante viscoso, puede deberse a los componentes del mismo Glyde $\AA$, el cual contiene EDTA al $15 \%$, peróxido de carbamida al $10 \%$ y excipientes como base para constituir el gel (Polietilenglicol-100, Propilenglicol, Pluronic F-127 y Alcohol cetílico). Esta base protege al EDTA de no ser oxidado por el peróxido, y además actúa como lubricante (Hülsmann et al., 2003). La presencia del peróxido en el quelante viscoso al ser usado en conjunción con $\mathrm{NaClO}$ es cuestionable, ya que al ser el hipoclorito un agente oxidante más potente que el peróxido, puede ser reducido por este agente y perder su eficacia. Es importante, tomar en cuenta que se observa un mayor efecto inhibitorio del hipoclorito de sodio, en cuanto a su capacidad para disolver tejido, cuando se usa de manera alternada con el peróxido, que cuando ambos se mezclan, (Hülsmann et al., 2003). Además, EDTA es capaz de inactivar al hipoclorito de sodio, pues es un agente reductor menos potente que el hipoclorito de sodio, así se une a este último y acaba con el cloro libre en solución. De esta manera, obstaculiza la capacidad de disolver tejidos del hipoclorito de sodio en solución (Grawehr et al.; Zehnder et al., 2005).

Las concentraciones de los irrigantes aplicadas en nuestro estudio son lo suficientemente activas como para generar una remoción eficiente del barro dentinario sin dañar de manera grave la arquitectura dentinaria (Schäfer \& Zapke, 2000; Calt \& Serper, 2002; Gambarini \& Laszkiewics, 2002; Hülsmann et al., 2005). Aunque en nuestra investigación la irrigación empleada fue $\mathrm{NaClO} 5,25 \%$ entre cada instrumento, e irrigación final con EDTA $17 \%$ por 1 minuto, luego $\mathrm{NaClO}$ y suero fisiológico, no se logró la completa eliminación del barro dentinario, al evaluarlo bajo microscopía óptica, esto al igual que estudios mencionados anteriormente (Hülsmann et al., 2002; Grandini et al.; Lim et al.; Prati et al.; Girard et al.; Dotto et al.; Yang et al.).

La presente investigación se basó en la herramienta utilizada por Lim et al., para la valoración del barro dentinario bajo microscopía óptica. Los demás estudios revisados lo evaluaban bajo microscopía electrónica de barrido, con el que obtenían observaciones más precisas de los túbulos dentinarios, restos pulpares, dentina intertubular y peritubular, etc.

Otra limitación que es importante manifestar, es la imposibilidad de conocer la edad cronológica de los dientes, o el tiempo transcurrido desde que fueron extraídos. Al no existir acceso a algún banco de dientes donde estuvieran clasificados, el grado de calcificación era azaroso entre las muestras del estudio, lo que pudo generar un nivel de desgaste dentinario diferente para cada espécimen, debido a que la acción de las sustancias quelantes depende del grado de calcificación de los conductos radiculares.

Por último, debido a que son innegables las ventajas que otorga Glyde $₫$ como lubricante para instrumentos rotatorios durante la instrumentación de conductos estrechos y curvados, su uso no debe descartarse, sino que debe seguir estudiándose hasta llegar a obtener resultados con alto nivel de evidencia, en cuanto a la totalidad de sus características. Lo anterior, puede ser logrado evaluando su empleo sólo en el primer instrumento utilizado para la preparación de los canales radiculares. Esto orientará la búsqueda de un protocolo de uso que aproveche sus excelentes características como lubricante.

AGRADECIMIENTOS: A la Dra. Rosa Guerra y al Dr. Ricardo Moreno por su ayuda y disposición en facilitarnos laboratorios para desarrollar la metodología de nuestra investigación.

ZAMORA, G.; FUENTES, R.; PESCHKE, E. \& NENEN, F. A Microscopic comparison of the residual smear layer remaining in root canals, after rotary instrumentation with and without a viscous chelator. Int. J. Odontostomat., 5(2):165-170, 2011.

ABSTRACT: Viscous chelators are used to help the instrumentation of narrow root canals, however, they could contribute to greater accumulated smear layer. The aim of this study was to compare the smear layer generated following instrumentation with or without the application of a viscous chelator. 39 inferior incisors were instrumented with Protaper® and divided into three groups. Irrigation in group A and B was performed after each instrument was changed, irrigation was realized as follows: EDTA $17 \%$ (1 minute), then $\mathrm{NaOCl} 5.25 \%$ and a final rinse with physiologic saline solution. Also in group A, a viscous chelator was used between each file. Group C irrigation was performed only with physiologic saline solution. Three teeth were not instrumented and irrigated (negative control). The roots were then longitudinally grooved and sectioned by thirds for microscopic evaluation. The detritus score obtained was compared statistically using non-parametric test $(p<0.05)$. In each third very low values of smear layer were found, and were the same in all three groups. In contrast, in the apical third of the groups $A$ and $C$ there was significantly more smear layer than in group B.

KEY WORDS: viscous chelator, smear layer, rotary instrumentation. 


\section{REFERENCIAS BIBLIOGRÁFICAS}

Baugh, D. \& Wallace, J. The role of apical instrumentation in root canal treatment: a review of the literature. J. Endod., 31:333-40, 2005.

Calt, S. \& Serper, A. Time-depend effects of EDTA on dentin structures. J. Endod., 28:17-9, 2002.

Dotto, S. R.; Travassos, R. M.; de Oliveira, E. P.; Machado, M. E. \& Martins, J. L. Evaluation of ethylenediaminetetraacetic acid (EDTA) solution and gel for smear layer removal. Aust. Endod. J., 33:62-5, 2007.

Gambarini, G. \& Laszkiewics, J. A scanning electron microscopic study of debris and smear layer remaining following use of GT rotary instruments. Int. Endod. J., 35:422-7, 2002.

George, S.; Kishen, A. \& Song, K. The role of environmental changes on monospecies biofilm formation on root canal wall by Enterococcus faecalis. J. Endod., 31:86772, 2005.

Girard, S.; Paqué, F.; Badertscher, M.; Sener, B. \& Zehnder, $M$. Assessment of a gel-type chelating preparation containing 1-hydroxyethylidene-1, 1-bisphosphonate. Int. Endod. J., 38:810-6, 2005.

Grandini, S.; Balleri, P. \& Ferrari, M. Evaluation of Glyde File Prep in combination with sodium hypochlorite as a root canal irrigant. J. Endod., 28:300-3, 2002.

Grawehr, M.; Sener, B.; Waltimo, T. \& Zehnder, M. Interactions of ethylenediamine tetraacetic acid with sodium hypochlorite in aqueous solutions. Int. Endod. J., 36:411-7, 2003.

Hülsmann, M.; Rümmelin, C. \& Schäfers, F. Root canal cleanliness after preparation with different endodontic handpieces and hand instruments: a comparative SEM investigation. J. Endod., 23:301-6, 1997.

Hülsmann, M.; Heckendorff, M. \& Schäfers, F. Comparative in-vitro evaluation of three chelator pastes. Int. Endod. J., 35:668-79, 2002.

Hülsmann, M.; Heckendorff, M. \& Lennon, A. Chelating agents in root canal treatment: mode of action and indications for their use. Int. Endod. J., 36:810-30, 2003.

Hülsmann, M.; Peters, O. \& Dummer, P. Mechanical preparation of root canals: shaping goals, techniques and means. Endodontic Topics, 10:30-76, 2005.
Jeon, I.; Spångberg, L.; Yoon, T.; Kazemi, R. \& Kum, K. Smear layer production by 3 rotary reamers with different cutting blade designs in straight root canals: a scanning electron microscopic study. Oral Surg. Oral Med. Oral Pathol. Oral Radiol. Endod., 96:601-7, 1997.

Lim, T.; Wee, T.; Choi, M.; Koh, W. \& Sae-Lim, V. Light and scanning electron microscopic evaluation of Glyde File Prep in smear layer removal. Int. Endod. J., 36:33643, 2003.

Naenni, N.; Thoma, K. \& Zehnder, M. Soft tissue dissolution capacity of currently used and potential endodontic irrigants. J. Endod., 30:785-7, 2003.

Prati, C.; Foschi, F.; Nucci, C.; Montebugnoli, L. \& Marchionni, S. Appearance of the root canal walls after preparation with NiTi rotary instruments: a comparative SEM investigation. Clin. Oral. Investig. 8(2):102-10, 2004.

Schäfer, E. \& Zapke, K. A comparative scanning electron microscopic investigation of the efficacy of manual and automated instrumentation of root canals. J. Endod., 26:660-4, 2000.

Shahravan, A.; Haghdoost, A.; Adl, A.; Rahimi, H. \& Shadifar F. Effect of smear layer on sealing ability of canal obturation: a systemtic review and meta-analysis. J. Endod., 33(2):96-105, 2007.

Yang, G.; Wu, H.; Zheng, Y.; Zhang, H.; Li, H. \& Zhou X. Scanning electron microscopic evaluation of debris and smear layer remaining following use of ProTaper and Hero Shaper instruments in combination with $\mathrm{NaOCl}$ and EDTA irrigation. Oral Surg. Oral Med. Oral Pathol. Oral Radiol. Endod., 106:63-71, 2008.

Zehnder, M.; Kosicki, D.; Luder, H.; Sener, B. \& Waltimo, T. Tissue-dissolving capacity and antibacterial effect of buffered and unbuffered hypochlorite solutions. Oral Surg. Oral Med. Oral Pathol. Oral Radiol. Endod., 94:756-62, 2002.

Zehnder, M.; Schmidlin, P.; Sener, B. \& Waltimo, T. Chelation in root canal therapy reconsidered. J. Endod., 31(11):817-20, 2005.

Dirección para correspondencia:

Gastón Zamora

Facultad de Odontología

Universidad de Valparaíso

Valparaíso

CHILE

Recibido : 15-07-2011

Aceptado: 28-07-2011 